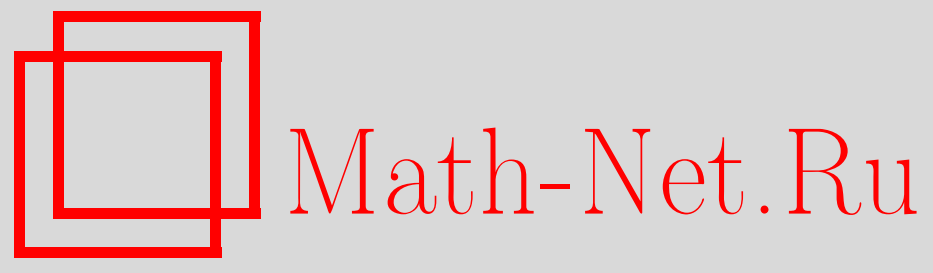

И. Н. Родионова, В. М. Долгополов, Задачи с сопряжением на характеристической плоскости для одного гиперболического уравнения третьего порядка в трехмерном пространстве, Вестн. Сам. гос. техн. ун-та. Сер. Физ.-мат. науки, 2014, выпуск 1(), 48-55

DOI: https://doi.org/10.14498/vsgtu1289

Использование Общероссийского математического портала MathNet.Ru подразумевает, что вы прочитали и согласны с пользовательским соглашением

http://www . mathnet.ru/rus/agreement

Параметры загрузки:

IP : 54.237 .59 .107

26 апреля 2023 г., 15:15:00

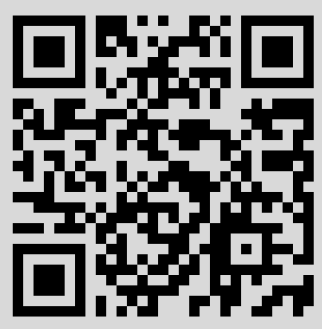


Вестн. Сам. гос. техн. ун-та. Сер. Физ.-мат. науки. 2014. № 1 (34). С. $48-55$

УДК 517.956.3

\title{
ЗАДАЧИ С СОПРЯЖЕНИЕМ НА ХАРАКТЕРИСТИЧЕСКОЙ ПЛОСКОСТИ ДЛЯ ОДНОГО ГИПЕРБОЛИЧЕСКОГО УРАВНЕНИЯ ТРЕТЬЕГО ПОРЯДКА В ТРЕХМЕРНОМ ПРОСТРАНСТВЕ
}

\author{
И. Н. Родионова, В. М. Долгополов
}

Самарский государственный университет,

Россия, 443011, Самара, ул. Академика Павлова, 1.

\begin{abstract}
Рассматривается полное уравнение гиперболического типа третъего порядка с переменными коэфбициентами в области, представляющей бесконечную треугольную призму, ограниченную характеристическими плоскостями $z=0$, $x=h$ данного уравнения $u$ двумя нехарактеристическими плоскостями $y=x$, $y=-x$. Решены две краевые задачи с данными на гранях призмы, являюшимися как характеристическими, так и нехарактеристическими плоскостями данного уравнения. В связи с трудностями склейки решений рассматриваемого типа гиперболических уравнений и заданием условий сопряжения на характеристике в условия сопряжения были введены интегралы и производные дробного порядка. На внутренней характеристической плоскости заданы условия сопряжения, содержащие производные дробного порядка искомой функиии, различные для обеих задач. Для данного уравнения авторами получено решение задачи Дарбу методом Римана, взятое за основу решения обеих поставленных задач, которые сводятся к однозначно разрешимым уравнениям Волътерры и Фредголъма соответственно, что позволило получить решения задач в явном виде.
\end{abstract}

Ключевые слова: интегралъные уравнения, краевые задачи, уравнения гиперболического типа высшего порядка.

Ряд краевых задач для уравнений гиперболического типа на плоскости и в пространстве требует склейки решения на характеристике данного уравнения. В связи с этим возникали трудности с заданием условий сопряжения на характеристике, т. к. традиционная склейка, содержащая нормальную производную искомого уравнения, зачастую приводила к некорректной постановке задачи. Поэтому в условия сопряжения на характеристике были введены интегралы и производные дробного порядка. Первые постановки таких задач принадлежат В. Ф. Волкодавову [1]. Затем они появлялись в ряде его работ с учениками [2-7].

Настоящая работа является продолжением исследований постановок и решений краевых задач для уравнений гиперболического типа в трехмерном пространстве, опубликованных в работах [8-10].

ISSN: 2310-7081 (online), 1991-8615 (print); doi: http://dx.doi.org/10.14498/vsgtu1289 (C) 2014 Самарский государственный технический университет.

Образец цитирования: И. Н. Р оди он ов а, В. М. Долгополо в, “Задачи с сопряжением на характеристической плоскости для одного гиперболического уравнения третьего порядка в трехмерном пространстве" // Вестн. Сам. гос. техн. ун-та. Сер. Физ.-мат. науки, 2014. № 1 (34). C. 48-55. doi: 10.14498/vsgtu1289.

Сведения об авторах: Ирина Николаевна Родионова (к.ф.-м.н., доц.), доцент, каф. математики и бизнес-информатики. Вячеслав Михайлович Долгополов (д.ф.-м.н., проф.), профессор, каф. математики и бизнес-информатики.

E-mail address: paskal1940@mail.ru (V.M. Dolgopolov, Corresponding author) 


\section{Рассмотрим уравнение}

$$
\begin{aligned}
L(u)=U_{x y z}+b(y) U_{x z}+ & a(x) U_{y z}+c(z) U_{x y}+b(y) c(z) U_{x}+ \\
& +a(x) c(z) U_{y}+a(x) b(y) U_{z}+a(x) b(y) c(z) U=0
\end{aligned}
$$

на множестве $H=H_{1} \cup H_{2}$,

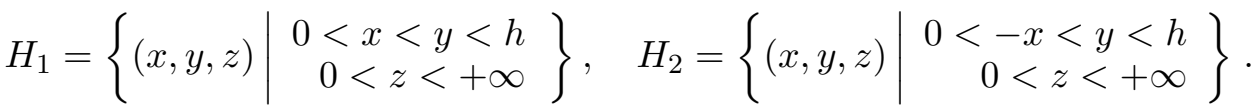

Функция $a(x)$ непрерывна на сегменте $[-h, h], b(y)$ - на сегменте $[0, h]$, $c(z)$ - на полуинтервале $[0,+\infty)$. Обозначим их первообразные соответственно $\alpha(x), \beta(y), \gamma(z)$.

ЗАДАчА I. На множестве $H$ найти решение уравнения (1), непрерывное в $\bar{H}$ и удовлетворяющее граничным условиям

$$
\begin{gathered}
U(x, x, z)=\tau_{1}(x, z), \quad(x, z) \in \bar{D}_{0}, \\
D_{0}=\{(x, z) \mid 0<x<h, 0<z<+\infty\}, \\
U(x,-x, z)=\tau_{2}(x, z), \quad(x, z) \in \bar{D}_{0}^{*}, \\
D_{0}^{*}=\{(x, z) \mid-h<x<0,0<z<+\infty\}, \\
U(x, y, 0)= \begin{cases}f_{1}(x, y), & (x, y) \in \bar{D}_{1}, D_{1}=\{(x, y) \mid 0<x<y<h\}, \\
f_{2}(x, y), & (x, y) \in \bar{D}_{2}, D_{2}=\{(x, y) \mid 0<-x<y<h\} ;\end{cases}
\end{gathered}
$$

а на плоскости $x=0-$ условиям сопряжения

$$
\begin{gathered}
\lim _{x \rightarrow 0+0} \frac{\partial}{\partial x} \int_{x}^{y} e^{\alpha(t)}(t-x)^{-r_{1}} U(t, y, z) d t=\delta_{1}(y), \\
\lim _{x \rightarrow 0-0} \int_{-y}^{x} e^{\alpha(t)}(x-t)^{-r_{2}} U(t, y, z) d t
\end{gathered}
$$

$\left(0<r_{1}, r_{2}<1, r_{1} \neq r_{2}\right)$.

ЗАДАчА II. На множестве $H$ найти решение уравнения (1), непрерывное в $\bar{H}$, с данными (4), а также

$$
U(x, h, z)= \begin{cases}\varphi_{1}(x, z), & (x, z) \in \bar{D}_{0} \\ \varphi_{2}(x, z), & (x, z) \in \bar{D}_{0}^{*}\end{cases}
$$

и условием сопряэсения на плоскости $x=0$

$$
\begin{array}{r}
\lim _{x \rightarrow 0+0}\left(\delta_{2}(y) \frac{\partial}{\partial x} \int_{x}^{h} e^{\beta(t)}(t-x)^{-r_{1}} U(x, t, z) d t-\frac{\partial}{\partial y}\left[e^{\beta(y)} U(x, y, z)\right]\right)= \\
=\lim _{x \rightarrow 0-0}\left(\delta_{3}(y) \frac{\partial}{\partial x} \int_{-x}^{h} e^{\beta(t)}(t+x)^{-r_{2}} U(x, t, z) d t+\frac{\partial}{\partial y}\left[e^{\beta(y)} U(x, y, z)\right]\right)+ \\
+g(y, z) .
\end{array}
$$

Для уравнения (1) задачи с сопряжением на характеристике рассматриваются впервые.

Для решения задачи I потребуем выполнения следующих условий. 
Условия A) $\tau_{1} \in C\left(\bar{D}_{0}\right), \tau_{1 x z}^{\prime \prime} \in C\left(D_{0}\right), \tau_{1}(x, 0)=\tau_{1}(0, z)=0, \tau_{2} \in C\left(\bar{D}_{0}^{*}\right)$, $\tau_{2 x z}^{\prime \prime} \in C\left(D_{0}^{*}\right), \tau_{2}(x, 0)=\tau_{2}(0, z)=0$; при $x=0 \tau_{1}$ и $\tau_{2}$ обращаются в нуль порядков выше $r_{1}$ и $r_{2}$ соответственно.

Функции $\varphi, \psi, f_{1}, f_{2}$ непрерывны в рассматриваемых областях вместе со своими смешанными частными производными второго порядка. Для них справедливо выполнение следующих условий.

Условия $\left.B_{1}\right) f_{i}(x, y) \in C\left(\bar{D}_{i}\right), f_{i x y}^{\prime \prime} \in C\left(D_{i}\right), i=1,2$.

Условия $\left.B_{2}\right) f_{1}(x, x)=0, f_{2}(x,-x)=0$; при $x=0 f_{i}(x, y)$ обращается в нуль порядка выше $r_{i}, i=1,2$.

Условие $\left.C_{1}\right) \delta_{1}(y) \in C^{(1)}[0, h]$.

Воспользуемся полученным в работе [10] методом Римана решением задачи Дарбу для уравнения (1), которое представимо в виде

$$
\begin{aligned}
U(x, y, z)=e^{\beta(x)-\beta(y)} & \tau_{1}(x, z)+ \\
& +\int_{x}^{y} N_{1}(t, z) e^{\alpha(t)+\beta(t)-\alpha(x)-\beta(y)} d t+e^{\gamma(0)-\gamma(z)} f_{1}(x, y)
\end{aligned}
$$

в области $H_{1}$ и

$$
\begin{aligned}
U(x, y, z)=e^{\beta(-x)-\beta(y)} \tau_{2}(x, z)+ & \\
& +\int_{-y}^{x} N_{2}(t, z) e^{\alpha(t)+\beta(-t)-\alpha(x)-\beta(y)} d t+e^{\gamma(0)-\gamma(z)} f_{2}(x, y)
\end{aligned}
$$

в области $H_{2}$.

Решение уравнения (1), определяемое формулами (8), (9), удовлетворяет условиям (2)-(4) задачи I. Неизвестные функции $N_{1}, N_{2}$ будем искать в классе функций, для которых выполняются следующие условия.

Условия $E) N_{1}(x, z), N_{2}(-x, z)$ непрерывны в $D_{0}$ вместе с частной производной по $z$, интегрируемы по $x$ на сегменте $[0, h]$ при любом $z \in[0,+\infty)$, $N_{i}(x, 0)=0, i=1,2$.

Из непрерывности решения на плоскости $x=0$ и условия сопряжения (5) получаем соотношения

$$
N_{1}(y, z) e^{\alpha(y)}=N_{2}(-y, z) e^{\alpha(-y)},
$$

$$
\begin{gathered}
-\int_{0}^{y} N_{1}(t, z) e^{\alpha(t)+\beta(t)} t^{-r_{1}} d t-e^{\alpha(y)+\beta(y)} y^{-r_{1}} \tau_{1}(y, z)+ \\
+\int_{0}^{y} \frac{\partial}{\partial t}\left[e^{\alpha(t)+\beta(t)} \tau_{1}(t, z)\right] t^{-r_{1}} d t= \\
=\delta_{1}(y)\left(\int_{0}^{y} N_{2}(-t, z) e^{\alpha(-t)+\beta(t)} t^{-r_{2}} d t+y^{-r_{2}} e^{\alpha(-y)+\beta(y)} \tau_{2}(-y, z)+\right. \\
\left.\quad+\int_{-y}^{0} \frac{\partial}{\partial t}\left[e^{\alpha(-t)+\beta(t)} \tau_{2}(t, z)\right](-t)^{-r_{2}} d t\right) .
\end{gathered}
$$


Отметим, что формула (11) получена при дополнительном ограничении, налагаемом на функции $f_{1}, f_{2}$ :

$$
\tau_{1} \int_{0}^{y} t^{-r_{1}-1} e^{\alpha(t)} f_{1}(t, y) d t+r_{2} \int_{0}^{y} t^{-r_{2}-1} e^{\alpha(t)} f_{2}(-t, y) d t=0
$$

которое обеспечивает выполнение равенства $N_{1}(x, 0)=N_{2}(x, 0)=0$.

После дифференцирования тождества (11) по у и некоторых преобразований с учётом равенства (10) приходим к интегральному уравнению относительно $N_{1}$ :

$$
\begin{aligned}
N_{1}(y, z) e^{\alpha(y)+\beta(y)}+\frac{\delta_{1}^{\prime}(y) y^{r_{1}+r_{2}}}{y^{r_{2}}+y^{r_{1}} \delta_{1}(y)} \int_{0}^{y} N_{1}(t, z) e^{\alpha(t)+\beta(t)} t^{-r_{2}} d t= \\
=\frac{F_{1}(y, z) y^{r_{1}+r_{2}}}{y^{r_{2}}+y^{r_{1}} \delta_{1}(y)}
\end{aligned}
$$

в котором

$$
\begin{aligned}
F_{1}(y, z)=r_{1} y^{-r_{1}-1} \tau_{1}(y, z) e^{\alpha(y)+\beta(y)} & +r_{2} y^{-r_{2}-1} \delta_{1}(y) \tau_{2}(-y, z) e^{\alpha(-y)+\beta(y)}+ \\
& +\delta_{1}^{\prime}(z) r_{2} \int_{0}^{y} e^{\alpha(-t)+\beta(t)} \tau_{2}(-t, z) t^{-r_{2}-1} d t
\end{aligned}
$$

Обозначим

$$
\omega(y)=\frac{y^{r_{1}}}{y^{r_{2}}+y^{r_{1}} \delta_{1}(y)} .
$$

Решение уравнения (13), полученное методом последовательных приближений, имеет вид

$$
\begin{aligned}
N_{1}(y, z) e^{\alpha(y)+\beta(y)}=-\delta_{1}^{\prime}(y) y^{r_{2}} \omega(y) & \int_{0}^{y} F_{1}(t, z) \omega(t) \times \\
& \times \exp \left[-\int_{t}^{y} \omega(u) \delta_{1}^{\prime}(u) d u\right] d t+F_{1}(y, z) y^{r_{2}} \omega(y) .
\end{aligned}
$$

При выполнении условий $\left.\left.\left.A), B_{1}\right), B_{2}\right), C_{1}\right)$ и равенства $(12)$ функции $N_{i}$ (в силу (10)) удовлетворяют условиям $E$ ). Подставляя найденные значения $N_{i}$ в формулы (8), (9), получаем решение задачи I в явном виде.

При решении задачи II будем предполагать выполнение условия $\left.B_{1}\right)$, а также следующие условия.

Условия $\left.B_{3}\right) f_{i}(x, h)=f_{i}(0, y)=\frac{\partial f_{i}(0, y)}{\partial x}=\frac{\partial f_{i}(0, y)}{\partial y}=0$

Условия $D) \varphi_{1}(x, z) \in C^{(1)}\left(\bar{D}_{0}\right), \frac{\partial \varphi_{1}(x, z)}{\partial x \partial z} \in C\left(D_{0}\right), \varphi_{2}(x, z) \in C^{(1)}\left(\bar{D}_{0}^{*}\right)$,

$$
\frac{\partial \varphi_{2}(x, z)}{\partial x \partial z} \in C\left(D_{0}^{*}\right), \varphi(0,0)=\varphi_{i x}^{\prime}(0,0)=0
$$

Условия $\left.C_{2}\right) \delta_{i}(y) \in C_{[0, h]}, i=2,3 ; g(y, z)$ непрерывна на множестве $0 \leqslant y \leqslant h$, $0 \leqslant z<+\infty$ и $g(y, 0)=0$. 
Для решения задачи II подчиним функции (8), (9) условиям (6), (7) соответственно и получим в области $H_{1}$

$$
\begin{aligned}
U(x, y, z)=\varphi_{1}(x, z) e^{\beta(h)-\beta(y)}- & \\
& -\int_{y}^{h} N_{1}(t, z) e^{\alpha(t)+\beta(t)-\alpha(x)-\beta(y)}+e^{\gamma(0)-\gamma(z)} f_{1}(x, y),
\end{aligned}
$$

в области $H_{2}$

$$
\begin{aligned}
U(x, y, z)=\varphi_{2}(x, z) e^{\beta(h)-\beta(y)}- & \\
& -\int_{y}^{h} N_{2}(t, z) e^{\alpha(-t)+\beta(t)-\alpha(x)-\beta(y)}+e^{\gamma(0)-\gamma(z)} f_{2}(x, y) .
\end{aligned}
$$

Непрерывность решения на плоскости $x=0$ приводит к соотношению (10), а условия сопряжения (7) - к равенству

$$
\begin{aligned}
& 2 \delta_{2}(y) \int_{0}^{h} N_{1}(t, z) e^{\alpha(t)+\beta(t)-\alpha(0)} K_{1}(t) d t- N_{1}(y, z) e^{\alpha(y)+\beta(y)-\alpha(0)}+F_{2}(y, z)= \\
&=-2 \delta_{3}(y) \int_{0}^{h} N_{2}(-t, z) e^{\alpha(-t)+\beta(t)-\alpha(0)} K_{2}(t) d t+ \\
&+N_{2}(-y, z) e^{\alpha(-y)+\beta(y)-\alpha(0)}, \quad(14)
\end{aligned}
$$

в котором введены обозначения

$$
\begin{gathered}
K_{1}(t)=\frac{1}{2}\left(\frac{\alpha^{\prime}(0) t^{1-r_{1}}}{1-r_{1}}+t^{-r_{1}}\right), \quad K_{2}(t)=-\frac{1}{2}\left(\frac{\alpha^{\prime}(0) t^{1-r_{2}}}{1-r_{2}}+t^{-r_{2}}\right), \\
F_{2}(y, z)=\left[C_{1} \varphi_{1 x}^{\prime}(0, z)+C_{2} \varphi_{1}(0, z)\right] \delta_{2}(y)- \\
-\left[C_{3} \varphi_{2 x}^{\prime}(0, z)+C_{4} \varphi_{2}(0, z)\right] \delta_{3}(y)-g(y, z),
\end{gathered}
$$

где

$$
C_{1}=\int_{0}^{h} e^{\beta(t)} t^{-r_{1}} d t, \quad C_{2}=\int_{0}^{h} e^{\beta(t)} \beta^{\prime}(t) t^{r_{1}} d t,
$$

а $C_{3}, C_{4}$ получаются из $C_{1}$ и $C_{2}$ заменой $r_{1}$ на $r_{2}$ соответственно.

Из соотношений $(10),(14)$ получаем интегральное уравнение относительно $N(y, z)=N_{1} e^{\alpha(y)+\beta(y)-\alpha(0)}:$

$$
N(y, z)=\int_{0}^{h} N(t, z)\left[K_{1}(t) \delta_{2}(y)+K_{2}(t) \delta_{3}(y)\right] d t+\frac{1}{2} F_{2}(y, z) .
$$

Так как свободный член уравнения непрерывен на множестве $0 \leqslant y \leqslant h$, $0 \leqslant z<+\infty$, что следует из условий $D)$ и $\left.C_{2}\right)$, функцию $N(y, z)$ будем искать в классе функций, удовлетворяющих условиям $E$ ), но условие интегрируемости на сегменте $[0, h]$ заменим условием непрерывности на указанном множестве. 
Как известно [11], интегральное уравнение Фредгольма второго рода с вырожденным ядром сводится к системе алгебраических уравнений. Обозначая

$$
A(z)=\int_{0}^{h} K_{1}(t) N(t, z) d t, \quad B(z)=\int_{0}^{h} K_{2}(t) N(t, z) d t
$$

получим

$$
N(y, z)=\delta_{3}(y) A(z)+\delta_{4}(y) B(z)+\frac{1}{2} F_{2}(y, z) .
$$

Умножая последовательно тождество (15) на $K_{1}(y)$ и $K_{2}(y)$ и интегрируя по сегменту $[0, h]$ обе части полученных равенств, получаем систему уравнений относительно $A(z)$ и $B(z)$ :

$$
A(z)\left(1-\int_{0}^{h} \delta_{2}(y) K_{1}(y) d y\right)-B(z) \int_{0}^{h} \delta_{3}(y) K_{1}(y) d y=\frac{1}{2} \int_{0}^{h} F_{2}(y, z) K_{1}(y) d y
$$

$-A(z) \int_{0}^{h} \delta_{2}(y) K_{2}(y) d y+B(z)\left(1-\int_{0}^{h} \delta_{3}(y) K_{2}(y) d y\right)=\frac{1}{2} \int_{0}^{h} F_{2}(y, z) K_{2}(y) d y$.

На функции $\delta_{2}, \delta_{3}$ наложим дополнительные ограничения в виде следующего условия.

Условие $\left.C_{3}\right) \delta_{2}$ и $\delta_{3}$ таковы, что

$$
\Delta=\left|\begin{array}{cc}
1-\int_{0}^{h} \delta_{2} K_{1} d y & -\int_{0}^{h} \delta_{3} K_{1} d y \\
-\int_{0}^{h} \delta_{2} K_{2} d y & 1-\int_{0}^{h} \delta_{3} K_{2} d y
\end{array}\right| \neq 0
$$

Найденные из системы (16) функции $A(z), B(z)$ подставим в равенство (15), получим с учётом (11) функции $N_{1}, N_{2}$, удовлетворяющие условию $E$ ) при выполнении условий $\left.\left.\left.\left.\left.B_{1}\right), B_{3}\right), D\right), C_{2}\right), C_{3}\right)$.

В заключение отметим, что единственность решения обеих задач следует из единственности решения методом Римана задачи Дарбу, взятой за основу, а также единственности решения интегральных уравнений, к которым свелись поставленные задачи.

Работа выполнена в рамках государственного задания высшим учебным заведениям в части проведения научно-исследовательских работ (1.909.2011).

This research was carried within the framework of the State Assignment to Universities on Participation in carrying out Scientific Research (1.909.2011).

\section{СПИСОК ЛИТЕРАТУРЫ/ REFERENCES}

1. В. Ф. Волкодавов, Е. И. Томина, О единственности решения ряда краевых задач для уравнения Лаврентвева-Бицадзе: Деп. в ВИНИТИ. 9.03.1993. 547-В93, 1993. [V. F. Volkodavov, E. I. Tomina, On the uniqueness of the solution for some boundary value problems of Lavrentiev-Bitsadze equation, Dep. VINITI. 9.03.1993. 547-B93, 1993 (In Russian)]. 
2. В. Ф. Волкодавов, С. Г. Маклаков, "Формула обращения для одного уравнения Вольтерра первого рода и ее применение"// Изв. вузов. Матем., 1996. №9. С. 16-20; V. F. Volkodavov, S. G. Maklakov, "An inversion formula for a Volterra equation of the first kind and its application", Russian Math. (Iz. VUZ), 1996, vol. 40, no. 9, pp. 14-17.

3. В. Ф. Волкодавов, В. Н. Захаров, “Экстремальные свойства решений одного уравнения гиперболического типа третьего порядка в трехмерном пространстве и их применение" // Изв. вузов. Матем., 1999. № 4. С. 28-31; V. F. Volkodavov, V. N. Zakharov, "Extremal properties of solutions of a third-order equation of hyperbolic type in three-dimensional space and their application", Russian Math. (Iz. VUZ), 1999, vol. 43, no. 4, pp. 26-29.

4. В. Ф. Волкодавов, Е. Р. Мансурова, "Краевая задача для частного вида уравнения Эйлера-Дарбу с интегральными условиями и специальными условиями сопряжения на характеристике" // Изв. вузов. Матем., 2000. № 8. С. 16-19; V. F. Volkodavov, E. R. Mansurova, "A boundary value problem for a particular form of the Euler-Darboux equation with integral conditions and special conjugation conditions on the characteristic", Russian Math. (Iz. VUZ), 2000, vol. 44, no. 8, pp. 14-17.

5. В. Ф. Волкодавов, Ю. А. Илюшина, “Для уравнения смешанного типа единственность решения задачи $T$ с сопряжением производной по нормали с дробной производной” // Изв. вузов. Матем., 2003. № 9. С. 6-9; V. F. Volkodavov, Yu. A. Il'yushina, "The solution of the problem $T$ with conjugation of the normal derivative with the fractional derivative is unique for an equation of mixed type", Russian Math. (Iz. VUZ), 2003, vol. 47, no. 9, pp. 4-7.

6. Е. Р. Мансурова, “Аналог задачи Трикоми с нелокальным интегральным условием сопряжения" // Изв. вузов. Матем., 2009. № 4. C. 61-66; E. R. Mansurova, "An analogue of the Tricomi problem with a nonlocal integral conjugation condition", Russian Math. (Iz. VUZ), 2009, vol.53, no. 4, pp. 49-53. doi: 10.3103/S1066369х09040094.

7. Е. Р. Мансурова, "Об однозначной разрешимости аналога задачи Трикоми с нелокальным интегральным условием сопряжения" // Матем. заметки, 2010. Т. 87, №6. С. 867876. doi: 10.4213/mzm6596; E. R. Mansurova, "On the unique solvability of an analogue of the Tricomi problem with a nonlocal integral conjugation condition", Math. Notes, 2010, vol. 87, no. 6, pp. 844-853. doi: 10.1134/S000143461005024X.

8. И. Н. Родионова, "Задача с интегральным условием для одного пространственного уравнения гиперболического типа, вырождающегося на координатных плоскостях" // Вестн. Сам. гос. техн. ун-та. Сер. Физ.-мат. науки, 2011. №2(23). С. 189-193. doi: 10.14498/vsgtu834. [I. N. Rodionova, "The problem with integral condition for one space analog of hyperbolic type equation degenerated on a coordinate planes", Vestn. Samar. Gos. Tekhn. Univ. Ser. Fiz.-Mat. Nauki, 2011, no. 2(23), pp. 189-193. (In Russian)].

9. М. В. Долгополов, И. Н. Родионова, "Задачи для уравнений гиперболического типа на плоскости и в трехмерном пространстве с условиями сопряжения на характеристике"// Изв. РАН. Сер. матем., 2011. Т. 75, №4. С. 21-28. doi: 10.4213/im4117; M. V. Dolgopolov, I. N. Rodionova, "Problems for equations of hyperbolic type in the plane or three-dimensional space with conjugation conditions on a characteristic", Izv. Math., 2011, vol. 75, no. 4, pp. 681-689. doi: 10.1070/IM2011v075n04ABEH002549.

10. В. М. Долгополов, И. Н. Родионова, "Две задачи для пространственного аналога гиперболического уравнения третьего порядка" // Вестн. Сам. гос. техн. ун-та. Сер. Физ.-мат. науки, 2012. № 4(29). C. 212-217. doi : 10.14498/vsgtu1114. [V. M. Dolgopolov, I. N. Rodionova, "Two problems for three-dimensional space analogue of the third order hyperbolic type equation", Vestn. Samar. Gos. Tekhn. Univ. Ser. Fiz.-Mat. Nauki, 2012, no. 4(29), pp. 212-217. (In Russian)].

11. С. Г. Михлин, Интегральные уравнения, М.-Л.: ОГИЗ, 1947. [S. G. Mikhlin, Integral'nye uravneniya [Integral Equations], Moscow, Leningrad, OGIZ, 1947 (In Russian)].

Поступила в редакцию 13/XI/2013; в окончательном варианте - 13/I/2014; принята в печать $-22 / \mathrm{I} / 2014$. 
MSC: 35L25; 35L35

\section{PROBLEMS WITH CONJUNCTION ON A CHARACTERISTIC PLANE FOR THE THIRD-ORDER HYPERBOLIC EQUATION IN THE THREE-DIMENSIONAL SPACE}

\section{N. Rodionova, V. M. Dolgopolov}

Samara State University,

1, Academician Pavlov st., Samara, 443011, Russian Federation.

In the present article the full equation of hyperbolic type of the third order with set of variable factors, in the area representing an infinite triangular prism, limited to the characteristic planes $z=0, x=h$ of the given equation and two noncharacteristic planes $y=x, y=-x$ is considered. Two boundary-value problems with data on the edges of the prism, which are both characteristic and non-characteristic planes of the given equation, are solved. In connection with difficulties of a gluing together of considered type solutions of the hyperbolic equations and the representation of conditions of interface on performance integrals and fractional derivatives have been introduced into interface conditions. On the interior characteristic plane the matching conditions, containing fractional order derivatives of required function, are established in order to avoid troubles with intersection of solutions. For equation considered in this article we have obtained the solution of the Darboux problem by method of Riemann, taken for the basis solutions of both problems, which are reduced to uniquely solvable equations of Volterra and Fredholm respectively, that has allowed to obtain the solutions of problems in the explicit analytic form.

Keywords: integral equations, boundary value problems, higher order hyperbolic type equations.

Received 13/XI/2013;

received in revised form $13 / \mathrm{I} / 2014$;

accepted $22 / \mathrm{I} / 2014$.

ISSN: 2310-7081 (online), 1991-8615 (print); doi: http://dx.doi.org/10.14498/vsgtu1289 (C) 2014 Samara State Technical University.

Citation: I. N. Rodionova, M. V. Dolgopolov, "Problems with conjunction on a characteristic plane for the third-order hyperbolic equation in the three-dimensional space", Vestn. Samar. Gos. Tekhn. Univ., Ser. Fiz.-Mat. Nauki [J. Samara State Tech. Univ., Ser. Phys. \& Math. Sci.], 2014, no. 1 (34), pp. 48-55. doi: 10.14498/vsgtu1289. (In Russian)

Authors Details: Irina N. Rodionova (Cand. Phys. \& Math. Sci.), Associate Professor, Dept. of Mathematics \& Business Informatics. Vyacheslav M. Dolgopolov (Dr. Phys. \& Math. Sci.), Professor, Dept. of Mathematics \& Business Informatics.

E-mail address: paskal1940@mail.ru (V.M. Dolgopolov, Corresponding author) 